\title{
Fast Feedback Systems for Orbit Correction in the TESLA Linear Collider
}

\author{
R.-D. Kohaupt, $\underline{\text { I. Reyzl }}$ *, DESY, Notkestr. 85, 22607 Hamburg, Germany
}

\section{Abstract}

In the TESLA Linear Collider feedback systems will provide an orbit correction within the bunch train. To limit the reduction in the targeted luminosity of $3.1 \cdot 10^{34} \mathrm{~cm}^{-2} \mathrm{~s}^{-1}$ due to beam separations to less than a few percent, the collisions have to be vertically stabilized within a fraction of the beam spot size of $5 \mathrm{~nm}$. One system, located in the beam delivery system (BDS), is used to correct vertical bunch offsets generated upstream in the main linear accelerator and BDS. The second at the interaction point (IP) brings the two beams into collision using the beam-beam deflection method. Both digital feedback systems provide a correction on a bunch-to-bunch basis. Their design is based on classical proportional-integral control (PI-control). This paper describes the feedback system designs. Results of simulations are presented.

\section{INTRODUCTION}

TESLA 500 is a conceptual design study of a future $\mathrm{e}^{+} \mathrm{e}^{-}$linear collider using superconducting $\mathrm{Nb}$ accelerating structures operating at $1.3 \mathrm{GHz}$ with a gradient of $25 \mathrm{MV} / \mathrm{m}$. TESLA is going to operate at a center of mass energy of $500 \mathrm{GeV}$ targeting a nominal luminosity of $3.1 \cdot 10^{34} \mathrm{~cm}^{-2} \mathrm{~s}^{-1}$ produced by head-on collision [1]. Reaching this goal requires beam spot sizes at the IP of $\sigma_{x}^{\star}=553 \mathrm{~nm}$ horizontally and $\sigma_{y}^{\star}=5 \mathrm{~nm}$ vertically. Each bunch train consists of 2820 bunches spaced by $337 \mathrm{~ns}$. The pulse repetition rate is $5 \mathrm{~Hz}$.

Due to the large vertical disruption parameter $D_{y}=33$ the luminosity is very sensitive to beam separations and crossing angles in the vertical plane, Fig.1. Limiting the maximum luminosity loss per bunch crossing to $10 \%$, two bunches have to interact within a separation of $5 \AA=$ $0.1 \sigma_{y}^{\star}$ and with a crossing angle smaller than $1.23 \mu \mathrm{rad}=$ $0.1 \sigma_{y}^{\star} ; \quad \sigma_{y^{\prime}}^{\star}$ denotes the natural divergence at the IP. Sources of undesired beam separations and crossing angles are e.g. Lorentz force detuning, wakefield effects and quadrupole movements or mechanical vibrations. The displacement of the two opposing final doublet magnets is a major concern, since a stationary final doublet displacement of $5 \mathrm{~nm}=1 \sigma_{y}^{\star}$ will half the luminosity. Simulations assuming large contributions of human produced noise to the ground motion spectrum predict a $1 \sigma_{y}^{\star}$ beam separation within $1 \mathrm{~ms}$ [3], which is roughly the bunch train length. From pulse to pulse the expected separation is at least $8 \sigma_{y}^{\star}$.

\footnotetext{
*Email: ingrid.reyzl@desy.de
}

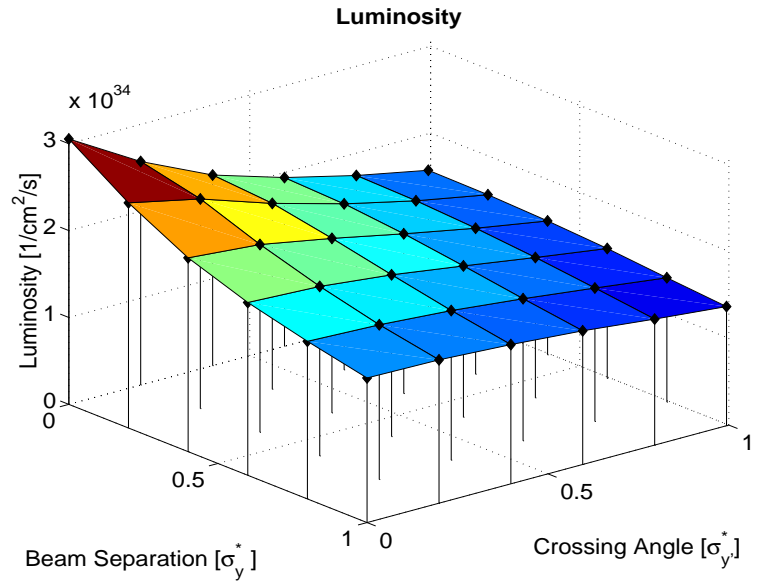

Figure 1: Luminosity as a function of beam separation and crossing angle. Data of surface plot are results of the program GUINEA PIG [6]

The size and the time scale of the vertical beam separation emphasize the necessity of a feedback system providing an intra-bunch train stabilization of the beam interaction at the IP. Due to the large bunch spacing of $337 \mathrm{~ns}$ a very beneficial orbit stabilization from one bunch to the next but one becomes feasible. In order to control the other beam phase a further feedback system will remove bunch offsets in the BDS within the bunch train. In this paper we describe the designs of these two digital feedback system using classical PI-control and present simulation results.

\section{FEEDBACK SYSTEM IN THE BDS}

The digital feedback system with a working frequency of $3 \mathrm{MHz}$ is located in the $30^{\circ}$ FODO-lattice of the tuning and diagnostic section in the BDS [2]. It provides a correction of bunch offsets in position and angle within the bunch train requiring a minimal feedback configuration of two beam position monitors (BPM) and two correctors. In order to correct offsets, bunch position measurements are taken with a resolution below $1 \mu \mathrm{m}$ [4] and transfered to a PI-controller determining the requested correction kicks. These kicks are then provided upstream by two correctors made up of fast kickers with a maximum kick of $0.12 \mu \mathrm{rad}$ and a rise time of $35 \mathrm{~ns}$ [7]. Since the processing time of BPM electronics incl. analog to digital conversion (ADC), of the controller and digital to analog conversion of its output signal, of amplifier and kicker and in addition the signal 
transmission time in the cables exceeds the bunch spacing of $337 \mathrm{~ns}$, a correction from one bunch to the second following bunch becomes feasible. The controller design ensures a reduction of a stationary bunch offset by two order of magnitudes after the $8^{\text {th }}$ bunch. Offsets below $157 \mathrm{kHz}$ are damped with $15 \mathrm{~dB}$ per decade.

After correction the beam trajectory can be distorted by displacement of magnets located in the final focus system. Quadrupole offsets will mainly lead to beam separations, since quadrupoles of large beta function are in phase with the final doublet. Beam separations created here will be then removed by the feedback system located at the IP.

\section{FEEDBACK SYSTEM AT THE IP}

In order to avoid serious luminosity loss a feedback system (working frequency $3 \mathrm{MHz}$ ) will stabilize the beam collision.It has to detect beam separations below the nanometer range, to steer the beams into collision, to complete the correction within a time which is much shorter than the bunch train length and to sustain the collision within a fraction of the vertical beam spot size. The design goal is a correction limiting the maximum luminosity loss to $10 \%$.

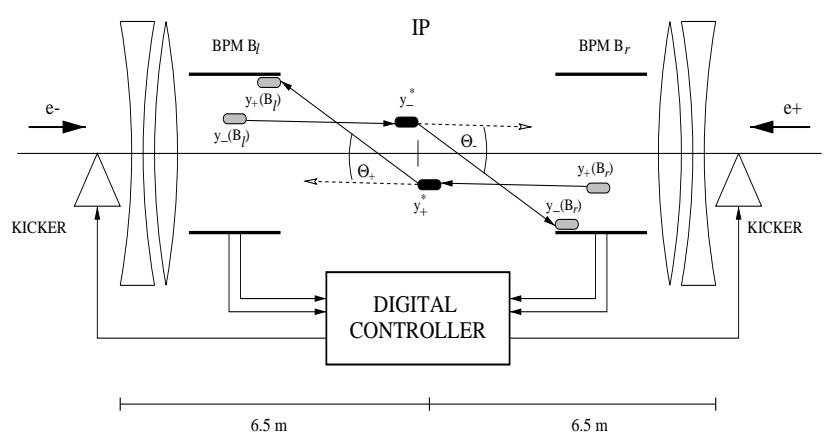

Figure 2: Scheme of the digital feedback system at the IP.

\subsection{Feedback Loop}

The feedback loop is characterized by four steps: detection of bunch separation, estimation of bunch separation, determination of correction kick by PI-controller and orbit correction of subsequent bunches by using fast kickers.

Detection of Bunch Separation The actual separation $\triangle y^{\star}$ between two bunches is given by

$$
\triangle y^{\star}:=y_{-}^{\star}-y_{+}^{\star}
$$

with $y_{-}^{\star}$ and $y_{+}^{\star}$ the $\mathrm{e}^{-}$and $\mathrm{e}^{+}$bunch positions at the IP.

Bunch separations become detectable by the beam-beam deflection caused by the attraction of the opposite charged bunches [5]. The experienced kick results in measurable position shifts of the outgoing bunches, Fig. 2. From the beam position measurements of the incoming and outgoing bunch of both beams at the two opposing final doublets the size and the sign of the bunch separation can be ascertained. We assume the $\mathrm{e}^{-}$bunch reaches the interaction region from the left, the $\mathrm{e}^{+}$bunch from the right side. The trajectory of the $\mathrm{e}^{-}\left(\mathrm{e}^{+}\right)$bunch between the left and the right BPM $B_{l}$ and $B_{r}$ is completely defined by its position $y_{-}^{\star}\left(y_{+}^{\star}\right)$ and angle $y_{-}^{\prime \star}\left(y_{+}^{\prime \star}\right)$ at the IP, the drift space $L=3 \mathrm{~m}$ between IP and BPMs together with the experienced beam-beam deflections $\theta_{-}\left(\theta_{+}\right.$, assumed to be $\left.-\theta_{-}\right)$. Including measurement errors $v$ and BPM misalignments $\delta$ the measurement of the incoming $\mathrm{e}^{-}$bunch at BPM $B_{l}$ is

$$
m_{-}\left(B_{l}\right)=y_{-}^{\star}-L y_{-}^{\star \star}+v_{-}\left(B_{l}\right)-\delta\left(B_{l}\right)
$$

and of the outgoing $\mathrm{e}^{-}$bunch at BPM $B_{r}$

$$
m_{-}\left(B_{r}\right)=y_{-}^{\star}+L\left(y_{-}^{\prime \star}+\theta\left(\triangle y^{\star}\right)\right)+v_{-}\left(B_{r}\right)-\delta\left(B_{r}\right) .
$$

The measurements $m_{+}\left(B_{r}\right)$ of the incoming $\mathrm{e}^{+}$bunch at the right BPM $B_{r}$ and $m_{+}\left(B_{l}\right)$ of outgoing $\mathrm{e}^{+}$bunch at the left BPM $B_{l}$ are defined accordingly. The combination of these four position measurements results in the starting equation for the orbit correction:

$$
\begin{aligned}
M & =2 \triangle y^{\star}+2 L \theta\left(\triangle y^{\star}\right)+V, \text { with } \\
M & :=m_{-}\left(B_{l}\right)-m_{+}\left(B_{l}\right)+m_{-}\left(B_{r}\right)-m_{+}\left(B_{r}\right), \\
V & :=v_{-}\left(B_{l}\right)-v_{+}\left(B_{l}\right)+v_{-}\left(B_{r}\right)-v_{+}\left(B_{r}\right) .
\end{aligned}
$$

Note, since only difference measurements are involved, the control does not depend on BPM misalignments $\delta\left(B_{l, r}\right)$.

Estimation of Bunch Separation Knowledge about the beam-beam deflection allows to find a suitable model needed by the controller to extract $\triangle y^{\star}$ from the BPM measurements. To receive an estimate $\triangle y_{F B}^{\star}$ of the bunch

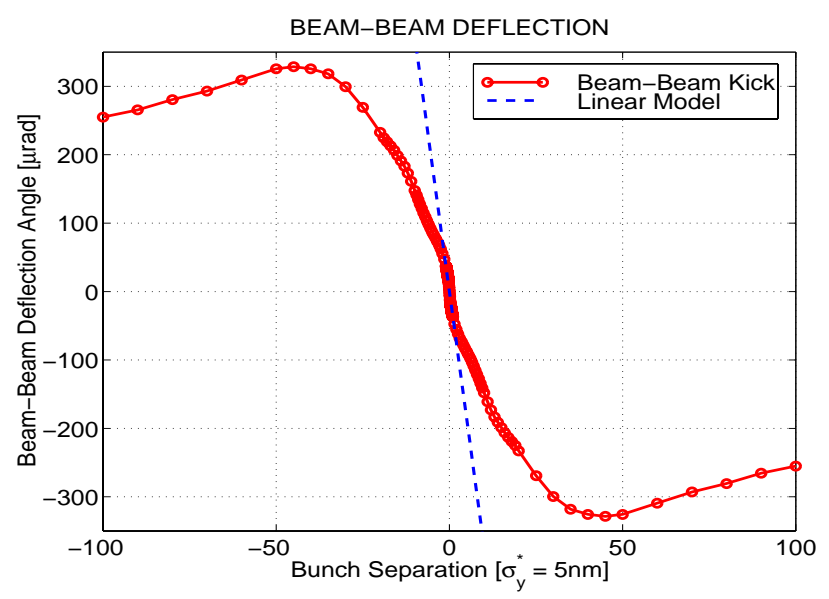

Figure 3: Beam-beam deflection angle $\theta$ vs. bunch separation; data are results of the program GUINEA PIG [6].

separation the beam-beam kick $\theta$ is substituted in Equ. 4 by its linear approximation $\theta^{F B}$, both plotted in Fig. 3:

$$
\theta^{F B}\left(\triangle y^{\star}\right)=-37.27 \frac{\triangle y^{\star}}{\sigma_{y}^{\star}} \quad[\mu \mathrm{rad}] .
$$

The slope of this linear approximation presents an essential value of the feedback loop: it determines how accurately the controller will determine a bunch separation in 
the nanometer range and by how much large separations will be underestimated. For example with the given linear model a bunch separation of $100 \sigma_{y}^{\star}$ is interpreted as $7 \sigma_{y}^{\star}$. The effect of this model error on the control is shown in 3.2 .

Determination and Provision of Correction The determination of the correction kick $u$ by the digital controller is based on the estimate bunch separations $\triangle y_{F B}^{\star}$. Implementing a PI-controller results in the control law

$$
u(k)=-K_{P} \triangle y_{F B}^{\star}(k)-K_{I} \sum_{j=0}^{k-1} \triangle y_{F B}^{\star}(j)
$$

where $k$ indicates the number of interaction (time step). $K_{P}$ and $K_{I}$ denote the gain of the proportional and integral controller. Their values define the feedback loop response.

Two kickers [7] with a rise time of $35 \mathrm{~ns}$ are placed on both sides of the IP, one meter upstream to the final doublet. They allow the coverage of a control range of $\pm 100 \sigma_{y}^{\star}$. The overall processing time of the feedback loop and the signal transmission time in a $50 \mathrm{~m}$ long cable insert a correction delay of two sample period.

\subsection{RESULTS}

Even so the linear model $\theta^{F B}$ used by the feedback system deviates severely from the non-linear beam-beam kick for large separations, large stationary offsets are successfully rejected. A constant beam separation of $100 \sigma_{y}^{\star}$ is reduced by 3 orders of magnitude already after 80 bunch interactions, emphasizing the robustness of the feedback system to model errors, Fig. 4. During correction the actual ver-

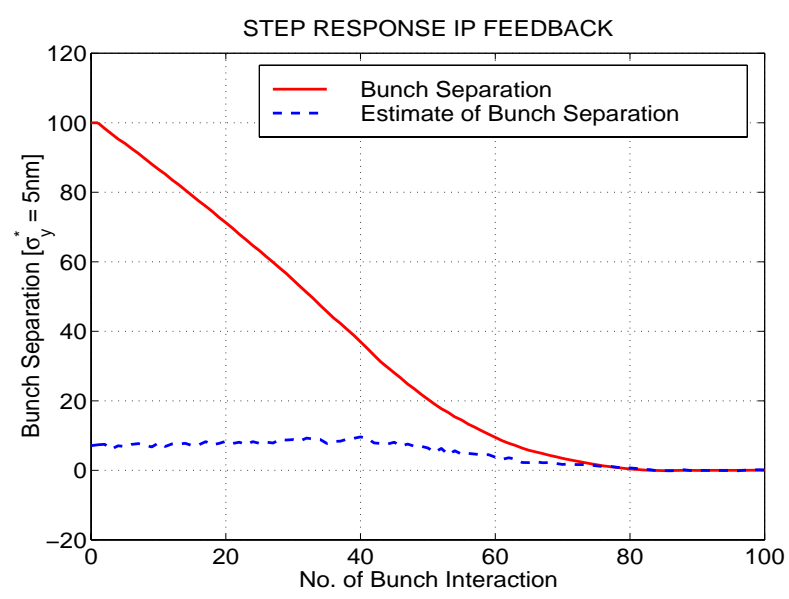

Figure 4: Response to a stationary $100 \sigma_{y}^{\star}$ beam separation.

tical separation is continuously underestimated. However, the feedback loop steers subsequent bunches in the right direction decreasing stepwise the occurring offset.

More realistic simulations include the bunch offsets at the linac exit. At the IP we further assume an rms BPM resolution of $5 \mu \mathrm{m}$ (add. noise), quantization errors caused by ADC, a $10 \%$ jitter of the beam-beam deflection angle due to bunch charge jitter (mult. noise) and kicker errors of $0.1 \%$ (mult. noise). Without the use of a feedback sys-

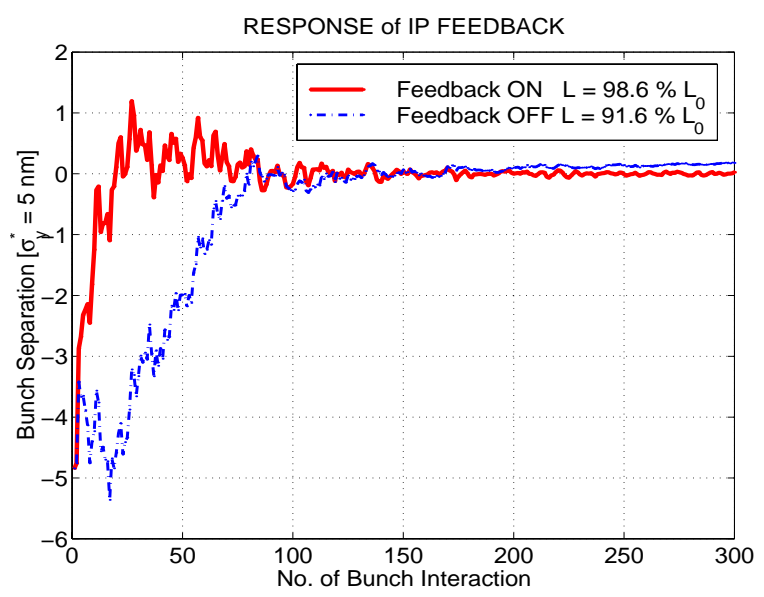

Figure 5: Response to beam separation including noise from the linac.

tem the luminosity is lowered by $8.4 \%$, whereas an active stabilization of the beam-interaction by a feedback system allows to achieve $98.6 \%$ of the nominal luminosity denoted by $L_{0}$, Fig. 5 .

Assuming an additional stationary beam separation of $50 \sigma_{y}^{\star}$ and $100 \sigma_{y}^{\star}$ caused by the displacement of the two final doublets, a luminosity of $95.6 \% L_{0}$ and $91.8 \% L_{0}$, respectively, becomes feasible. Limiting the maximum luminosity loss by $10 \%$, the use of the IP-feedback system thus relaxes the rms displacement tolerance of pulse-topulse jitter of the final doublet magnets to $200 \mathrm{~nm}$.

\section{ACKNOWLEDGMENTS}

It is a pleasure for us to thank our colleagues at DESY.

\section{REFERENCES}

[1] R. Brinkmann, High Luminosity with TESLA 500, DESY, TESLA 97-13, 1997

[2] I. Reyzl, Feasibility Study of Feedback Systems for Orbit Correction in the TESLA, Linear Collider, Shaker Verlag, Aachen, 1999

[3] R. Brinkmann, G. Materlik, J. Rossbach, A. Wagner, Conceptual Design of a $500 \mathrm{GeV} e^{+} e^{-}$Linear Collider with Integrated X-ray Laser Facility, DESY 1997-048, 1997

[4] R. Lorenz: Cavity Beam Position Monitors, AIP Conference Proceedings 451, p. 53-73, 1999

[5] P. Bambade, R. Erickson, Beam-Beam Deflection as an Interaction Point Diagnostic for the SLC, SLAC-PUB 3979, 1986

[6] D. Schulte, Study of Electromagnetic and Hadronic Background in the Interaction Region of the TESLA Collider, TESLA 97-08, 1997

[7] J. Rümmler, Feedback Kickers in the DESY Rings, IEEE Conference Proc. of the EPAC 94, London, England, 1994 